\section{EFICIÊNCIA RELATIVA DE EMPRESAS DO SETOR ATACADISTA/DISTRIBUIDOR NO BRASIL}

Ana Paula Souza* Oscar Tupy**

\section{Introdução}

Atualmente, existem 2.288 empresas no setor atacadista/distribuidor e 1.057 empresas prestadoras de serviços. Essas empresas comercializam produtos alimentícios industrializados, candies, bebidas, produtos de higiene pessoal, limpeza doméstica, produtos farmacêuticos, perfumaria, papelaria, material de construção, entre outros. Dentro desse perfil de atuação, o setor como um todo faturou, em 2004, R\$ 76,5 bilhões a preço de varejo, o que representa $55,6 \%$ do mercado, gerando mais de 143 mil empregos diretos, com uma equipe superior a 65 mil representantes comerciais e 48 mil veículos (entre próprios e terceirizados). O faturamento do setor deve-se ao resultado da atuação das forças de vendas, sendo 15,2\% de vendas diretas, 59,3\% indiretas e o restante compondo-se de vendas de auto-serviço e vendas efetuadas por meio de telemarketing.

Segundo a Associação Brasileira de Distribuição - ABAD (2005), o setor atacadista/distribuidor compõe-se de empresas que percorrem o país e abastecem a população, enfrentando constantes desafios e dificuldades, tais como: estradas precárias, riscos de assaltos e roubos de cargas, alto custo de manutenção de frotas, perdas, altos custos operacionais, créditos restritos e o eterno peso da carga tributária, entre outros.

São 900 mil pontos-de-venda, em todo o país; mais da metade é de pequeno porte e depende das empresas atacadistas/distribuidoras, uma vez que a indústria não pode atendê-los diretamente. Para atender as necessidades do

* Graduada em Economia e aluna do curso de especialização Lato Sensu em Finanças Empresariais do Centro Universitário de Araraquara - Uniara.

** Professor do Mestrado em Desenvolvimento Regional e Meio Ambiente do Centro Universitário de Araraquara - Uniara e membro do Grupo de Pesquisa Multidisciplinar "Gestão das Organizações”, da Uniara. Pesquisador Embrapa Pecuária Sudeste. E-mail: tupy@cppse.embrapa.br. pequeno varejo, as empresas atacadistas/distribuidoras adotaram novos e modernos procedimentos na compra e venda de mercadorias, investindo em tecnologia, valorizando informações para atender a evolução do mercado, possibilitando como resultado a diversificação de produtos adequados, preparando o pequeno varejista para o mercado competitivo, oferecendo-lhes inclusive diversas condições de financiamento, entre outros serviços (ABAD, 2005).

Segundo a ACNIELSEN (2004), o setor encerrou o ano de 2004 com $55,4 \%$ do mercado de abastecimento. Mesmo em 2003, um período ruim para a economia, quando o faturamento do setor de alimentos caiu, o atacado conseguiu crescer em relação a 2002. Esse crescimento ocorreu devido ao aumento de produtividade e fortalecimento do pequeno varejo. As empresas que passaram pelos anos 80, período de inflação elevada, e que conseguiram sobreviver aos primeiros anos do Plano Real, quando muitos associaram o atacado à imagem de estoques especulativos, investiram em logística, melhoraram a tecnologia interna e o controle dos negócios, conseguindo reestruturar-se no mercado.

Embora seja um período muito curto (1994 a 2004), insuficiente para tirar da memória as más práticas do passado, em que o lucro era obtido inclusive pela retenção dos estoques, esse crescimento no setor nos últimos anos devese à reordenação do processo de cadeia produtiva (ABAD, 2005).

As empresas atacadistas/distribuidoras têm como meta sempre atender a um aumento da demanda e vender mais, com investimentos em novas áreas de armazenagem, melhorando o desempenho no atendimento aos pequenos varejistas.

Segundo Barrizzelli (2005), o setor de distribuição está mais forte e organizado, visando cada vez mais cumprir um papel importante no mercado de consumo. Mas a eficiência da cadeia de abastecimento depende da indústria, pois esta é quem decide como quer que sua marca chegue ao ponto-de-venda. A estratégia de uma empresa para estimular pedidos é determinada pelo alto investimento em marketing, que para melhor resultado, depende exclusivamente da distribuição física (entrega pontual), sabendo-se que a probabilidade do produto não estar nas gôndolas no momento certo é muito grande.

Para a ABAD (2005), a indústria sempre espera que os atacadistas se superem, ou seja, que além de distribuírem seus produtos, sejam capazes de efetuar trocas de produtos, que façam ponto-de-venda e avaliem o mix do varejista; porém se o atacadista assim o fizer, além de vender, distribuir, adicionar serviços de troca de mercadorias e arrumação de gôndola, vai se transformar gradativamente em um distribuidor, que só atende um número limitado de fabricantes. 
Segundo Barrizzelli (2005), o setor passou por três fases distintas em relação às vendas. O primeiro corresponde ao período de 1991 a 1996, época em que a atividade cresceu acentuadamente, cerca de $41,6 \%$, sobre uma base relativamente fraca; o segundo, de 1996 a 2001, foi marcado pela estabilidade, quando a variação entre o maior e o menor valor foi de apenas $2,8 \%$, refletindo o contexto macroeconômico desse período; já o terceiro, de 2002 a 2004, é marcado pela volta de crescimento. Nesses dois últimos anos, o setor apresentou um crescimento de $31,4 \%$

Durante a era inflacionária, a indústria viabilizava seu negócio nas operações de compra e venda, e o atacadista trabalhava com oportunidades. Com a estabilidade econômica, teve-se a necessidade de mudanças nos procedimentos de trabalho, assim o atacadista passou a ser um prestador de serviços de distribuição. Hoje, o objetivo das empresas não é mais armazenar mercadorias e lucrar com vendas de produtos, mas, sim, agregar serviço e distribuição.

Como elo estratégico da cadeia de suprimentos, a distribuição está passando por rápidas transformações no Brasil. Nos últimos dez anos, o setor esteve motivado por duas grandes frentes com as quais está igualmente ligado. Por um lado, a expansão das grandes redes supermercadistas e as ações de pequenos e médios varejistas em busca de maior competitividade vêm alterando a estrutura da distribuição do país. De outro lado, a indústria fornecedora, em busca de maior capilaridade de alcance dos seus produtos, investe e passa a exigir parceiros com nível de desenvolvimento tecnológico.

Atento a esses dois pólos e, principalmente, às mudanças nos hábitos de consumo dos brasileiros, o segmento atacadista/distribuidor aperfeiçoa seus modelos de atuação para se adaptar às mudanças impostas pela organização, reduzindo custos fixos e aumentando a eficiência na prestação de serviços, principalmente a pequenos varejistas (ABRAS, 2004).

Dada a importância do setor atacadista/distribuidor para a economia, influenciando os preços no varejo, entende-se como necessário avaliar a eficiência relativa das suas empresas no Brasil, sendo que os resultados poderão contribuir para a gestão empresarial, elevando-se o desempenho do setor e reduzindo custos para o varejo em geral.

Para que os empresários possam idealizar um programa de melhoria nos níveis das operações das empresas e saber se o mesmo será possível de executar, torna-se fundamental avaliar o seu desempenho produtivo. A urgência, direção e prioridades de melhoramento serão determinadas parcialmente pela identificação do desempenho atual da operação. Portanto, todas as operações produtivas precisam, de alguma forma, de uma medida de desempenho como um pré-requisito para o seu melhoramento.
Nesta perspectiva, o trabalho avalia a eficiência relativa (técnica e de escala) de uma amostra das empresas que compõem o setor de distribuição no Brasil.

\section{Metodologia}

\section{O modelo empírico de análise}

Neste trabalho, foi utilizado, para medir a eficiência relativa das empresas, o método de programação matemática Data Envelopment Analysis (DEA), descrito por COELLI (1994). Sendo que a solução do problema é dada pela seguinte formulação:

\section{$\operatorname{Min} \theta, \lambda \theta$}

Sujeito a:

$$
\begin{gathered}
-y i+Y \lambda \geq 0, \\
\theta x i-x \lambda \geq 0, \\
\lambda \geq 0,
\end{gathered}
$$

Onde è é um escalar, cujo valor será a medida de eficiência da i-ésima firma e o parâmetro ë é um vetor Nx1, cujos valores são calculados de forma a obter a solução ótima. Para uma firma eficiente, todos os valores ë serão zero; para uma firma ineficiente, os valores de ë serão os pesos utilizados na combinação linear de outras firmas eficientes, que influenciam a projeção da firma ineficiente sobre a fronteira calculada. Isso significa que, para uma unidade eficiente, os pesos calculados fornecerão a firma virtual da firma ineficiente, mediante combinação linear. As unidades eficientes que, quando combinadas, fornecem a firma virtual para a firma ineficiente são conhecidas como peers (pares) ou benchmarks (firmas eficientes, de melhor prática) para aquela firma (GOMES, 1999).

O valor de è obtido será o escore de eficiência para i-ésima firma e a condição de que $0<$ è $<1$ será satisfeita, com o valor de 1 indicando um ponto na fronteira e, portanto, uma firma eficiente. Note que o problema de programação linear deve ser resolvido $\mathrm{N}$ vezes, uma para cada firma da amostra.

A medida de eficiência obtida da equação (3) é orientada para os insumos, pressupondo retornos constantes de escala (RC) para a tecnologia.

Além da pressuposição de RC, podem-se considerar retornos variáveis de escala (RV) para a tecnologia. A pressuposição de RC somente é apropriada quando todas as firmas estão operando em uma escala ótima. Deste modo, o problema de programação linear com retornos constantes de escala, conforme representado na equação (3), pode ser facilmente modificado para atender a 
pressuposição de RV pela adição de uma restrição de convexidade: N1'ë= 1, conforme demonstrado na equação (4):

\section{$\operatorname{Min} \theta, \lambda \theta$}

Sujeito a:

$$
\begin{gathered}
-y i+y \lambda \geq 0, \\
\theta x i-x \lambda \geq 0, \\
N 1^{\prime}=1 \\
\lambda \geq 0
\end{gathered}
$$

onde N1 é um vetor N x 1 de uns. Esta restrição forma um casco convexo que "envelopa" os pontos de forma mais ajustada do que o casco cônico formado pela restrição de RC e, portanto, provê escores de eficiência técnica que são maiores ou iguais àqueles obtidos, usando o modelo com RC. A restrição de convexidade (N1'ë=1) assegura, essencialmente, que a firma ineficiente é somente comparada com uma firma de igual tamanho. O ponto projetado para a firma sobre a fronteira DEA será uma combinação convexa de firmas observadas. Portanto, no caso DEA com RC, a firma pode ser comparada com firmas que são substancialmente maiores (menores) do que ela (COELLI, 1994). Neste caso, os pesos ë poderão somar um valor maior do que 1 .

Dada a pressuposição de RV para a tecnologia, os escores de eficiência técnica obtidos sob a pressuposição de RC podem ser decompostos em dois componentes, um devido à ineficiência de escala e um devido à ineficiência técnica pura, como apresentado nas equações (5) e (6) a seguir:

$$
\text { ETrC }=\text { ETrV } \times \text { EesC }
$$$$
\text { Eesc }=\text { Etrce } / \text { ETRV }
$$

\section{Onde,}

ETrce = é a eficiência técnica obtida sob a pressuposição de RC;

$\mathrm{ETrv}$ = é a eficiência técnica obtida sob a pressuposição de RV;

Eesc = é a eficiência de escala.

Contudo, a medida de eficiência de escala obtida da equação (6) não indica se a firma está operando em uma área de retornos crescentes ou decrescentes de escala. Este problema pode ser contornado resolvendo um problema DEA adicional com retornos não-decrescentes à escala impostos. Para tanto, altera-se a equação (4), substituindo a restrição N1'ë=1 por N 1'ë? 1, obtendo:

$\operatorname{Min} \theta, \lambda \theta$

Sujeito a:

$$
\begin{gathered}
-y i+y \lambda \geq 0, \\
\theta x i \quad-x \lambda \leq 1 \\
N 1^{\prime} \leq 1 \\
\lambda \geq 0,
\end{gathered}
$$

Os retornos crescentes e decrescentes de escala são calculados considerando a diferença entre os escores de eficiência técnica obtidos pela solução do problema DEA da equação (4) e aqueles obtidos pela solução do problema DEA da equação (5). Escores iguais indicam firmas operando com retornos decrescentes de escala e ao contrário, com retornos crescentes. Para mais detalhes, consultar Coelli (1994).

A restrição N1'ë<1 assegura que i-ésima firma não seja comparada com firmas substancialmente maiores do que ela, mas pode ser comparada com firmas menores.

\section{Dados}

Os dados utilizados neste trabalho foram obtidos da Revista da ABAD setor de distribuição, Ranking 2005. Procurou-se avaliar a eficiência relativa apenas das empresas cuja modalidade de operação é a entrega, uma vez que o trabalho não teve a pretensão de esgotar o assunto. Além disso, segundo os dados publicados pela ABAD (2005), em parceria com a ACNIELSEN e a FIA (Fundação Instituto de Administração), a modalidade mais utilizada é de entrega que, no ano de 2004, atendeu 73,9\% dos varejistas. Enquanto que atendimento de Balcão é o menos utilizado, atendendo apenas 3,3\%, no ano de 2004.

As variáveis de decisão utilizadas para a construção da fronteira de eficiência foram o faturamento (FAT), área de armazenagem em metros quadrados (ARM), números de funcionários (NFUNC), força de vendas - FVE ( $\mathrm{n}^{\mathrm{o}}$ de vendedores diretos e representantes), total de veículos da frota transportadora (TF) e $\mathrm{n}^{\circ}$ de clientes ativos (NCA).

\section{Procedimentos para calcular os escores de eficiência do setor atacadista/ distribuidor}

As soluções dos problemas de programação linear das equações 3, 4 e 5 forneceram os escores de eficiência neste trabalho. Nas referidas equações, $X$ é a matriz de fatores de produção (área de armazenagem, números de funcionários, força de vendas, total de veículos) e $\mathrm{n}^{\circ}$ de clientes ativos, de 
dimensões ( $\mathrm{K} \times \mathrm{N}$ ); e $\mathrm{Y}$ o vetor de produtos (faturamento das empresas) de dimensões $(\mathrm{Mx} \mathrm{N})$, representando os dados de todas as empresas de abastecimento da amostra. Tem-se, ainda, xi o vetor coluna de insumos e yi o vetor coluna de produtos representando a i-ésima empresa. As letras gregas è e ë foram definidas acima.

O programa utilizado para implementar as soluções dos problemas de programação linear foi o Data Envelopment Analysis Program (DEAP), desenvolvido por Coelli (1994).

\section{Resultado e Discussão}

Estimativa da eficiência técnica de empresas do setor atacadista/ distribuidor no Brasil.

As estatísticas descritivas das variáveis utilizadas no modelo constam do Quadro 1, a seguir:

Quadro 1 - Estatística descritiva dos dados do setor de atacado/distribuidor $(\mathrm{n}=137)$.

\begin{tabular}{|l|c|c|c|c|c|c|c|c|}
\hline & ETRV & EESC & FAT (RS MIL) & NCA & ARM (M⿻ ${ }^{2}$ ) & NFC & FVE & FTO \\
\hline MÉDIA & 0,663 & 0,746 & $38.372,73$ & $5.057,03$ & $4.955,66$ & 100,42 & 62,42 & 40,891 \\
\hline D.PADRÃO & 0,226 & 0,263 & $64.496,07$ & $6.903,92$ & $17.205,44$ & 185,51 & 110,07 & 70,916 \\
\hline MÁXIMO & 1,000 & 1,000 & $416.750,89$ & $55.100,00$ & $200.000,00$ & $1.500,00$ & 945,00 & 604,000 \\
\hline MíNIMO & 0,286 & 0,102 & $1.200,00$ & 300,00 & 100,00 & 4,00 & 3,00 & 1,000 \\
\hline
\end{tabular}

Fonte: Dados da pesquisa.

As estatísticas descritivas dos dados apresentadas no Quadro 1 mostram as médias, desvio padrão, valor máximo e mínimo para as variáveis, faturamento, número de clientes ativos, área de armazenagem, número de funcionários, número de vendedores diretos e representantes (força de venda) e veículos da frota.

As estimativas de eficiência relativa das empresas atacadistas/ distribuidoras constam do Quadro 2, dado a seguir:
Quadro 2 - Sumário de eficiência técnica e de escala de empresas do setor atacadista/distribuidor no Brasil.

\begin{tabular}{|c|c|c|c|c|c|}
\hline Obs. & Razão Social & UF & ETRV & EESC & RET \\
\hline 1 & Zamboni Distribuidora Ltda. & RJ & 1,000 & 1,000 & $\mathrm{C}$ \\
\hline 2 & União Com. Imp. e Exp. Ltda. & MG & 0,950 & 0,699 & $\mathrm{D}$ \\
\hline 3 & Universe Distribuidora Ltda. & MG & 1,000 & 1,000 & $\mathrm{C}$ \\
\hline 4 & Alimentos Zaeli Ltda. & PR & 0,466 & 0,834 & D \\
\hline 5 & Pastifício Santa Amália Ltda. & MG & 0,549 & 0,672 & $\mathrm{D}$ \\
\hline 6 & Garcia Atacadista Ltda. & $\mathrm{RJ}$ & 1,000 & 1,000 & \\
\hline 7 & Distribuidora Zangirolami Ltda. & SP & 0,551 & 0,974 & D \\
\hline 8 & Real Distr. Log. Ltda. & $\mathrm{GO}$ & 1,000 & 1,000 & $\mathrm{C}$ \\
\hline 9 & Sucessores de Dorival Ribeiro Ltda. & PR & 0,991 & 0,836 & D \\
\hline 10 & Asa Distribuidora e Rep. Com. Ltda. & MG & 0,922 & 1,000 & $\mathrm{C}$ \\
\hline 11 & Cbs. Alimentos Ltda. & RS & 0,744 & 1,000 & $\mathrm{C}$ \\
\hline 12 & Frohlich S/A Ind. Com. Cereais & RS & 0,551 & 0,984 & D \\
\hline 13 & Distri. Alim. Francisco Ikeda Ltda. & SC & 0,669 & 0,973 & $\mathrm{D}$ \\
\hline 14 & Karnekeijo Ind. Com. Imp. Exp. Ltda. & $\mathrm{PE}$ & 0,818 & 0,999 & I \\
\hline 15 & Distribuidora Internacional de Alim. & RN & 0,675 & 0,990 & I \\
\hline 16 & Linhares Diesel Ltda. & ES & 1,000 & 1,000 & $\mathrm{C}$ \\
\hline 17 & Ludovico J. Tozzo Ltda. & $\mathrm{SC}$ & 0,646 & 0,933 & D \\
\hline 18 & Unilíder Distribuidora Ltda. & ES & 0,727 & 0,965 & D \\
\hline 19 & Júlio Stampa Distribuidor Ltda. & PR & 1,000 & 1,000 & $\mathrm{C}$ \\
\hline 20 & Atende Logística S/A & MG & 0,759 & 1,000 & $\mathrm{C}$ \\
\hline 21 & Paulicéa Com. Repres. Ltda. & RJ & 0,424 & 0,994 & I \\
\hline 22 & Scapol Dist. e Prod. Higiene Ltda. & SP & 1,000 & 1,000 & $\mathrm{C}$ \\
\hline 23 & Jotujé Distribuidora Ltda. & $\mathrm{CE}$ & 0,598 & 0,988 & I \\
\hline 24 & Nordesa Com. e Rep. Ltda. & PB & 0,886 & 0,999 & I \\
\hline 25 & Distribuidora de Alimentos Prado & RS & 0,826 & 0,964 & D \\
\hline 26 & Crlcrn Distr. Prod. Aliment. Ltda. & SP & 0,521 & 0,998 & I \\
\hline 27 & Com. e Repres. Bornholdt Ltda. & MS & 0,524 & 0,998 & I \\
\hline 28 & Novas Amazonas Ind. Com. Imp. Ltda. & $\mathrm{DF}$ & 0,475 & 0,999 & $\mathrm{C}$ \\
\hline 29 & Disploki Distr. Com. Repr. Ltda. & SP & 0,542 & 0,938 & D \\
\hline 30 & Stival Alimentos Ind. e Com. Ltda. & PR & 0,507 & 0,988 & D \\
\hline 31 & Atacadão Com. de Gên. Alim. Ltda. & RS & 1,000 & 1,000 & $\mathrm{C}$ \\
\hline 32 & Pararela Distr. de Alim. e Repr. Ltda. & BA & 0,374 & 0,986 & I \\
\hline 33 & Copralon Cial. Prod. Alim. Londrina & PR & 0,790 & 0,881 & $\mathrm{D}$ \\
\hline 34 & Distribuidora de Alim. Mirassol Ltda. & $\mathrm{BA}$ & 0,846 & 0,983 & \\
\hline 35 & Mix Distribuidor Ltda. & $\mathrm{BA}$ & 0,782 & 0,954 & \\
\hline 36 & Nilo Tozzo\& Cia. Ltda. & $\mathrm{SC}$ & 0,502 & 0,976 & \\
\hline
\end{tabular}




\begin{tabular}{|c|c|c|c|c|c|}
\hline 37 & Pedro de Oliveira & MA & 0,809 & 0,997 & I \\
\hline 38 & Disjoi Dist. Log./Sc & $\mathrm{SC}$ & 0,577 & 0,994 & I \\
\hline 39 & Royal de Campo Grande Dist. Ltda. & RJ & 0,612 & 0,970 & I \\
\hline 40 & Marbel Rc Com. Imp. e Exp. Ltda. & $\mathrm{PE}$ & 0,630 & 0,793 & I \\
\hline 41 & Del-Distribuidora Cardeal Ltda. & PI & 0,618 & 0,911 & I \\
\hline 42 & Eliseu Martins Distr. Rep. Ltda. & RS & 0,414 & 0,956 & I \\
\hline 43 & Dec Sul Ltda. & SC & 0,438 & 0,998 & I \\
\hline 44 & Riomeo Distribuição Ltda. & PR & 0,606 & 0,966 & I \\
\hline 45 & Belpar Distribuidora de Cosméticos Ltda. & $\mathrm{PE}$ & 0,601 & 0,916 & I \\
\hline 46 & Disbombons Dist. de Bombons Ltda. & PB & 0,441 & 0,951 & I \\
\hline 47 & Elson's Produtos Alim. Ltda. & ES & 0,368 & 0,782 & I \\
\hline 48 & Ariovaldo Costa Paulo \& Cia Ltda. & PR & 0,296 & 0,868 & I \\
\hline 49 & G.Costa- Distr de Alimentos & MG & 0,574 & 0,880 & I \\
\hline 50 & Pacaluz Com. e Log. Ltda. & $\mathrm{SC}$ & 0,624 & 0,885 & I \\
\hline 51 & Distribuidora Condor Ltda. & $\mathrm{CE}$ & 0,383 & 0,905 & I \\
\hline 52 & Multigiro Distr. Repres. Ltda. & PR & 0,607 & 0,912 & I \\
\hline 53 & Disjoi. Dist.Log/Rs & RS & 0,286 & 0,998 & I \\
\hline 54 & Boulevard Distribuidora Ltda. & $\mathrm{AM}$ & 0,553 & 0,731 & I \\
\hline 55 & Vallette Distr. Prod Ltda. & DF & 0,477 & 0,867 & I \\
\hline 56 & Carlos Gilmar Caxias da Costa & $\mathrm{BA}$ & 0,912 & 0,887 & I \\
\hline 57 & Logistica Distribuidora Ltda. & $\mathrm{SC}$ & 0,392 & 0,862 & I \\
\hline 58 & Muller Novo Horizonte Distr. Ltda. & SC & 0,466 & 0,887 & I \\
\hline 59 & Comeia Distribuidora Ltda. & BA & 0,444 & 0,840 & \\
\hline 60 & Ada Distribuidora Ltda. & PE & 0,497 & 0,984 & I \\
\hline 61 & Tiscoski Distribuidora & SC & 1,000 & 0,938 & I \\
\hline 62 & Cacaus Distribuidora Ltda. & PR & 0,296 & 0,872 & I \\
\hline 63 & Altamir Mateus Brai do \& Cia Ltda. & RS & 1,000 & 1,000 & $\mathrm{C}$ \\
\hline 64 & Distrinorte Dist. Prod. Alim. & MG & 0,293 & 0,924 & I \\
\hline 65 & Central Sr Dist. Prod. Alim. Ltda. & RS & 0,538 & 0,930 & \\
\hline 66 & Acre Caxias Ltda. & RS & 1,000 & 1,000 & $\mathrm{C}$ \\
\hline 67 & Dampp Perfumes e Cosméticos Ltda. & GO & 0,554 & 0,937 & I \\
\hline 68 & Comercial Roma Ltda. & $\mathrm{CE}$ & 0,830 & 0,581 & I \\
\hline 69 & Brassol Brasília Alim. Sorv. Ltda. & DF & 0,456 & 0,815 & I \\
\hline 70 & Mika da Amazônia Alimentos Ltda. & MT & 0,445 & 0,741 & I \\
\hline 71 & Centro Distr. Alim. e Limp. Ltda. & BA & 0,358 & 0,796 & \\
\hline 72 & Distr. de Cosméticos Santos Ltda. & $\mathrm{BA}$ & 0,761 & 0,831 & I \\
\hline 73 & Polina e Polina Dist. Alim. Ltda. & RS & 0,701 & 0,888 & I \\
\hline 74 & Distribuidora Cristal Ltda. & PI & 0,349 & 0,780 & \\
\hline 75 & Andrade \& Bergton Ltda & $\mathrm{BA}$ & 0,458 & 0,736 & I \\
\hline 76 & Multibom Distribuidora de Prod. Alim. Ltda. & $\mathrm{SC}$ & 0,659 & 0,719 & I \\
\hline 77 & Gibahia Comercial Ltda. & $\mathrm{BA}$ & 0,311 & 0,801 & I \\
\hline 78 & Marcas Premium Log. Dist. Ltda. & BA & 0,377 & 0,823 & I \\
\hline 79 & Wat Alimentos Ltda. & SP & 0,558 & 0,784 & I \\
\hline 80 & Moinho Brasília Ltda. & GO & 0,454 & 0,711 & I \\
\hline 81 & Acrojohn Distr. da Amazônia Ltda. & $\mathrm{AM}$ & 0,792 & 0,848 & I \\
\hline 82 & Brago Com. Rep. Prod. Químicos Ltda. & DF & 0,660 & 0,569 & I \\
\hline 83 & Araújo Mateus Imp. e Com. Ltda. & BA & 0,985 & 0,872 & I \\
\hline 84 & Comercial Rofe Ltda. & MA & 0,330 & 0,893 & I \\
\hline 85 & Promix Distr. Prod. Hig. Limpeza Ltda. & SP & 0,651 & 0,921 & I \\
\hline 86 & Polimarcas Distribuidora Ltda. & $\mathrm{SP}$ & 1,000 & 1,000 & $\mathrm{C}$ \\
\hline 87 & Comercial de Alimentos Caio Ltda. & DF & 0,688 & 0,654 & I \\
\hline 88 & Frios Vilhena Impot. e Export. Ltda. & $\mathrm{AC}$ & 0,699 & 0,602 & I \\
\hline 89 & Da\& A Distribuição Ltda. & $\mathrm{PE}$ & 1,000 & 1,000 & $\mathrm{C}$ \\
\hline 90 & Bernutri Distribuição Ltda. & SP & 0,382 & 0,787 & I \\
\hline
\end{tabular}

\begin{tabular}{|c|c|c|c|c|c|}
\hline 91 & Clonex Produtos e Sist. Limpeza Ltda. & RS & 0,301 & 0,778 & I \\
\hline 92 & Cooper Distribuidora Ltda. & $\mathrm{SC}$ & 0,565 & 0,632 & I \\
\hline 93 & Redijohn Dist. Prod. Hig. Limp. Ltda. & PE & 0,495 & 0,721 & I \\
\hline 94 & Timoneiro Dist. de Alim.Ltda. & $\mathrm{SC}$ & 1,000 & 1,000 & $\mathrm{C}$ \\
\hline 95 & Takigawa Comércio de Frios Ltda. & $\mathrm{AC}$ & 0,822 & 0,489 & I \\
\hline 96 & Realpet Distr. Ltda. & $\mathrm{SC}$ & 0,819 & 0,748 & I \\
\hline 97 & Bigolin Dist. de Alimentos Ltda. & $\mathrm{SC}$ & 0,418 & 0,886 & I \\
\hline 98 & Inbahia Representações e Distrib. Ltda. & $\mathrm{BA}$ & 0,489 & 0,600 & I \\
\hline 99 & Expansão Com.\& Repes. Ltda. & PI & 0,359 & 0,665 & I \\
\hline 100 & Comércio de Gen.Alim.Wk. Ltda. & MT & 0,489 & 0,415 & I \\
\hline 101 & Zmg Rep. Prod. Higiênicos & RS & 0,411 & 0,628 & I \\
\hline 102 & Belmax Comercial Ltda. & ES & 0,733 & 0,499 & I \\
\hline 103 & Comercial Coutrin Ltda. & $\mathrm{BA}$ & 0,393 & 0,439 & I \\
\hline 104 & Flf Comércio Ltda. & SE & 0,448 & 0,518 & I \\
\hline 105 & Elson'sdistribuidora Ltda. & $\mathrm{BA}$ & 0,468 & 0,452 & I \\
\hline 106 & E.A. Friedrich\& Cia. Ltda. & RS & 0,781 & 0,553 & I \\
\hline 107 & Joaci Santana Garcia & RS & 1,000 & 0,915 & I \\
\hline 108 & Agro Veterinária Timbo & $\mathrm{SC}$ & 1,000 & 0,813 & I \\
\hline 109 & Centro Norte Distribuidora Ltda. & $\mathrm{AC}$ & 0,727 & 0,414 & I \\
\hline 110 & Sul Real Dist. Prod. Alim. Ltda. & RS & 0,722 & 0,505 & I \\
\hline 111 & Acrojohn Distrib. da Amazônia Ltda. & RR & 1,000 & 0,333 & I \\
\hline 112 & Comercial Arruda Ltda. & $\mathrm{AL}$ & 0,868 & 0,331 & I \\
\hline 113 & Comercial Zip Ltda. & $\mathrm{PE}$ & 0,618 & 0,316 & I \\
\hline 114 & Zaira Rezende Silva Capiberibe & MS & 0,314 & 0,592 & I \\
\hline 115 & Triunfo Distribuidora Ltda. & MS & 0,397 & 0,472 & I \\
\hline 116 & Gimabel Comercial Ltda. & $\mathrm{PE}$ & 0,580 & 0,348 & I \\
\hline 117 & L\&L Logistica Ltda. & PI & 1,000 & 0,365 & I \\
\hline 118 & Barcelo Distribuidora Ltda. & SP & 0,756 & 0,291 & I \\
\hline 119 & Confortin Com. Representações & $\mathrm{SC}$ & 0,956 & 0,420 & \\
\hline 120 & Júlio César Parnoff & $\mathrm{SC}$ & 1,000 & 0,443 & I \\
\hline 121 & Nutrimentos do Vale S. Franscico Co. & PE & 0,937 & 0,386 & I \\
\hline 122 & J.A. Calheiros de Melo Jr. & $\mathrm{PE}$ & 0,867 & 0,371 & I \\
\hline 123 & Distribuidora Hass Ltda. & $\mathrm{SC}$ & 0,502 & 0,342 & I \\
\hline 124 & Cdn- Centro de Distribuição do Ne Ltda. & $\mathrm{AL}$ & 0,636 & 0,405 & I \\
\hline 125 & José Alves Distribuidora Ltda. & PI & 1,000 & 0,478 & I \\
\hline 126 & Distribuidora de Laticínios do Ampá Ltda. & $\mathrm{AP}$ & 0,651 & 0,304 & I \\
\hline 127 & S\&M Distribuidora Ltda. & $\mathrm{BA}$ & 0,621 & 0,285 & I \\
\hline 128 & Comercial Gasperazzo & ES & 0,551 & 0,329 & I \\
\hline 129 & Potência Distribuidora Ltda. & $\mathrm{SC}$ & 0,524 & 0,259 & I \\
\hline 130 & Globo Dist. Brokerlog. Ltda. & SE & 0,784 & 0,254 & I \\
\hline 131 & Wsd Com.de Alimentos Ltda. & MT & 0,575 & 0,227 & I \\
\hline 132 & Distr. Pedro Schneider Ltda. & $\mathrm{SC}$ & 1,000 & 0,291 & I \\
\hline 133 & Comercial Poligrãos Ltda. & $\mathrm{BA}$ & 1,000 & 0,203 & I \\
\hline 134 & Distribuidora Schulz Ltda. & $\mathrm{SC}$ & 1,000 & 0,159 & I \\
\hline 135 & Cavallini Rep. Com. Distr. Ltda. & $\mathrm{BA}$ & 0,709 & \begin{tabular}{l|l}
0,102 & \\
\end{tabular} & I \\
\hline 136 & Superacre Import. Export. Ltda. & $\mathrm{AC}$ & 1,000 & 0,109 & I \\
\hline 137 & Assis e Borges Ltda. & RR & 0,701 & 0,144 & I \\
\hline \multicolumn{3}{|c|}{ Média } & 0,663 & 0,746 & \\
\hline
\end{tabular}

$\mathrm{C}=$ retorno constante de escala; $\mathrm{D}$ = retorno decrescente de escala; $\mathrm{I}$ = retorno crescente de escala. Fonte: Dados de Pesquisa. 
A eficiência técnica média, pressupondo retornos variáveis de escala para a amostra, foi de 66,3\%, significando que, em média, as empresas da amostra estão utilizando 33,7 \% mais fatores de produção (área de armazenagem, números de funcionários, força de vendas, veículos) e atendendo mais clientes do que necessitam para o nível de faturamento realizado.

Pode-se verificar, neste estudo, que, das empresas relacionadas, 24 obtiveram níveis de eficiência máxima $q=1,000$, e estão definindo a fronteira para as empresas menos eficientes desta amostra, podendo ser consideradas referência para outras empresas.

A eficiência de escala média das empresas da amostra foi de 74,6\%. Do total, 13 empresas operaram a custos crescentes, uma vez que se localizaram na faixa de retornos decrescentes da fronteira. Na faixa de retornos constantes, localizaram-se 17 empresas e as demais, na faixa de retornos crescentes. Se adotarem um tamanho mais produtivo, as empresas poderão, em média, elevar de 25,4 \% a sua eficiência produtiva.

Empresas eficientes tecnicamente, mas que apresentam ineficiência de escala, deverão adotar estratégias distintas em função da sua localização, se na faixa de retornos crescentes ou decrescentes de escala. Empresas que estão operando na faixa de retornos crescentes deverão explorar os benefícios e aumentar a produção por meio da expansão dos insumos, uma vez que aumentos proporcionais nestes resultam em aumentos mais do que proporcionais no produto (faturamento). Contudo, empresas que estão operando na faixa de retornos decrescentes deverão ajustar os seus ativos (armazéns, frota) e mãode-obra até que passem a operar na faixa de retornos constantes de escala, uma vez que retornos decrescentes de escala indicam que aumentos proporcionais nos insumos resultam em aumentos menos do que proporcionais do produto (faturamento). As estratégias para as empresas ineficientes tecnicamente e em escala também serão distintas em função dos retornos de escala serem crescentes ou decrescentes. Empresas que estão operando na faixa de retornos crescentes deverão aumentar o faturamento, sem, contudo, aumentar os insumos, e empresas que estão operando na faixa de retornos decrescentes deverão ajustar os seus ativos até o limite da eficiência técnica máxima e, posteriormente, proceder ajustes adicionais nestes, até que passem a operar na faixa de retornos constantes de escala.

\section{Conclusões e recomendações}

O setor atacadista/distribuidor, desde o seu surgimento até os dias atuais, passou por vários processos de transformações que permitiram sua evolução; investindo em modernização e tecnologia e aumentando a capilaridade de entrega. Adotaram estratégias para aproximar-se do cliente, antecipando suas necessidades, melhorando o mix de produtos e sua performance em 2004 em relação a anos anteriores. Contudo, a eficiência técnica média de 66,3 \%, aqui obtida, para as empresas do setor (modalidade entrega), indica um excesso de gastos no processo de distribuição (área, mão-de-obra, força de venda, frota e clientes) dado o faturamento obtido.

Os níveis de eficiência estimados para as empresas do setor atacadista/ distribuidor, que fizeram parte deste trabalho, indicam que há muito a ser feito para que o setor seja eficiente na distribuição. O nível de eficiência estimado pode onerar toda a cadeia de suprimentos e principalmente o pequeno varejo. As empresas do setor precisam estar atentas aos níveis de eficiência aqui obtidos, procurando, se possível, identificar os seus fatores determinantes. Fatores como logística de distribuição, qualidade e nível de treinamento da mão-de-obra, qualidade e manutenção da frota, deverão certamente ser levados em conta, como também as estratégias de relação com a indústria e o varejo (clientes). Outros fatores residuais de períodos de elevada inflação, como armazéns muito grandes, assim como o péssimo estado das rodovias brasileiras, não podem ser desconsiderados. Contudo, recomenda-se a realização de estudos mais abrangentes para o setor, se possível, parcerias entre a ABAD e institutos de pesquisa.

\section{Referências:}

ASSOCIAÇÃO BRASILEIRA DE DISTRIBUIÇÃO - ABAD. Revista Distribuição. Ranking 2005. Disponível em: <www.abad.com.br>. Acesso em: 10/09/2005.

ASSOCIAÇÃO BRASILEIRA DE SUPERMERCADOS - ABRAS: banco de dados, 2004. Disponível em: <www.abras.com.br>. Acesso em: 10/09/ 2005.

BARRIZZELLI, N. Oportunidades à vista. Associação Brasileira de Distribuição - ABAD. Revista Distribuição, São Paulo, Ano XIII, no 147, p.18- 24, 2005.

COELLI, T. J. A Guide to DEAP Version 2.1: A Data Envelopment Analysis (Computer) Program. Department of Econometrics, University of New England, Armidale, 1994. Mimeo.

GOMES, A.P. Impactos das transformações da produção de leite no número de produtores e requerimentos de mão-de-obra e capital. Tese (Doutorado em Economia Rural). 1999. Universidade Federal de Viçosa UFV. Viçosa-MG. 1999.157p. 


\section{Resumo:}

O setor atacadista/distribuidor tem apresentado atualmente um considerável crescimento, devido ao aumento dos pequenos varejos espalhados por todo o país. Introduzido pelos imigrantes europeus, mais especificamente os portugueses, na década de 50, o setor atacadista /distribuidor fortaleceu-se na década de 90 , passando por três fases distintas em relação às vendas. A primeira corresponde ao período de 1991 a 1996, época em que a atividade cresceu, significativamente, cerca de $41 \%$, reagindo positivamente à transição e valorização da moeda nacional; a segunda foi de 1996 a 2001, marcada pela estabilidade; já a terceira, de 2002 a 2004, evidencia-se pela volta de crescimento de 31,4\%. Este trabalho refere-se a uma análise da eficiência relativa (técnica e de escala) de uma amostra de empresas do setor atacadista /distribuidor no Brasil, utilizando-se dados publicados na Revista Distribuição - Ranking 2005. A eficiência relativa foi avaliada pelo método Data Envelopment Analysis DEA. Os resultados do trabalho mostraram que o nível de eficiência técnica foi em média $66,3 \%$, sinalizando às empresas a necessidade de reavaliar cuidadosamente as suas estratégias de gestão, uma vez que, níveis elevados de ineficiência técnica significam uso em excesso de ativos, cujos custos poderão, se repassados ao varejo, elevar os preços dos produtos. Além disso, empresas ineficientes são menos competitivas no mercado.

Palavras-chave:

Setor Atacadista/Distribuidor, Eficiência Produtiva, Eficiência Técnica. 